\title{
Multi-scale analysis of jack pine saplings after fire across burn severities
}

\author{
Michael Bomber ${ }^{1}$, Raechel Portelli ${ }^{1}$ \\ ${ }^{1}$ Department of Geography, Environment, and Spatial Science, Michigan State University, East Lansing, MI, corresponding: \\ raechel@msu.edu
}

\section{Commission III}

KEY WORDS: Multiscale analysis, UAS, forest recovery, machine learning

\begin{abstract}
:
Fire serves as a successional initiation in jack pine (Pinus banksiana) forests of North America, as jack pine reproduce using seratonous cones that open only in intense heat. Jack pine seedling resilience after fire is characterized by high numbers of mortality. The estimation of sapling survivability and density is useful for understanding dynamics of carbon sequestration, forest structure and dynamic, and supporting management of the landscape. Most studies concerning the interaction of forest disturbances occurs at moderate spatial resolution. These moderate resolution data analyses do not adequately capture the fine scale spatial variation of the landscape after fire for understanding sapling survival. Thus, high-resolution data, such as aerial photography may provide more detailed information to support decision-making. A key to the types of spatial patterns that emerge in these early years is the pre-fire stand condition. In heavily managed areas, the mosaic of forest patches may include extensive variety in disturbance conditions. In this current research we address the problem of scale in relation to understanding the influence of prefire condition on post-fire early recovery patterns. To do this, we combine data output from the LandTrendr algorithm in Google Earth Engine with spectral data from aerial photography collected by airplane and Unmanned Aerial System to perform a random forest classification. The result is a finer scale resolution map of forest conditions of varying sapling density.
\end{abstract}

\section{INTRODUCTION}

Fire disturbance is a major influence on the pattern for northern conifer forests. Understanding the recovery process is key to studying global carbon cycling, forest dynamics, and informing forest management decision-making (Meng et al.). Vegetation recovery is impacted not only by burn severity but also by prefire forest structure and condition (Fernandez-Manso et al.). For some tree species, such as jack pine (Pinus banksiana) high temperature, typically in the form of fire, is key to its reproduction.

While jack pine stands are more resilient that mixed conifer stands to fire, fire activity still leads to extensive changes in forest age and structure, especially in young tree stands (Tucker and Kashian). These young forest stands (age $<10$ years) are characterized by sparse forest canopy cover and diverse grasslike vegetation. Such habitats become home to threatened species like the Kirtland Warbler and the Upland Sandpiper (Corace et al.; Spaulding and Rothstein).

The use of spectral vegetation indices such as Normalized Burn Ratio (NBR) and Normalized Vegetation Difference Index (NDVI) are commonly applied to moderate resolution remote sensing data, such as Landsat, for monitoring forest disturbance and recovery (Frazier et al.). Over the past decade, these data have led to the development of robust systems for detection forest disturbance and recovery. For example, the LandTrendr tool, developed by (Kennedy et al.) provides users with the ability to map spectral index change from 1984 to present. Such information provides essential information about the spatiotemporal patterns activated across the landscape. However, the use of such coarse resolution imagery makes it difficult to characterize short term local changes in forest structure due to the small size of young trees and the spectral noise arising from background vegetation.

High-resolution remote sensing data in the form of aerial photography has long been used by forest managers for decision-making (Lydersen and Collins). The National Aerial Imagery Program, operated by the U.S. Department of Agriculture provides digital orthophotography at various spatial (1-2m), spectral (RGB or RGB+NIR) and temporal scales (5-yr repeat, 2003-2008 ; 3-yr repeat, 2008-present). Additionally, Unmanned Aerial Systems (UAS) have become a reliable means for collecting high-resolution RGB+NIR imagery for forest applications (Matese)

The integration of high-resolution imagery in the form of aerial photography (NAIP and UAS) provides a means for better mapping early successional forests. When combined with coarser resolution data, such as Landsat, these data may lead to more detailed models of the spatiotemporal change at short-term scales. The goal of this work was to develop a working model for integrating Landsat, UAS, and NAIP imagery for mapping variations in jack pine sapling presence across different disturbance histories.

\subsection{Background}

Post-fire vegetation recovery is a key successional stage where new vegetation becomes a carbon sink, offsetting fire-induced carbon loss (Hicke et al.). Recovering landscapes exhibit unique patterns based on burn severity, local topography, and vegetation. Local variations lead to heterogeneous seedling establishment and success. For forest managers, sapling success is key to cost-savings and estimation of future forest density. 
Jack pine re-establishment is influenced by abiotic (e.g., elevation, temperature, localized precipitation, burn severity) and biotic factors (e.g., plant species and pre-fire stand age) interacting to determine the patterns of sapling density. Previous studies of regeneration have utilized LiDAR and multispectral aerial photographs to characterize sapling densities, for example (Potter et al.).

Unmanned Aerial Systems (UAS) provide a means for mapping post-fire vegetation with multispectral data (Pádua et al.). In these studies, NBR and NDVI are effective for timely data acquisition. The inclusion of geometric data either using LIDAR or Structure-from-Motion (SfM) can improve these estimates (Meng et al.). However, LIDAR data are not always available for wildland sites, while SfM can be time intensive due to the extent of image overlap for photogrammetric reconstruction.

When coupled with GEOBIA spatial analysis methods can be implemented, leading to more robust insights about local processes Previous research indicated that the use of GEOBIAUAS approaches were consistently accurate in the location of jack pine saplings (redacted). A related study in dry ecosystems also found UAS to be sufficient in identifying young lavender plants (Buters et al.). Spatial location alone, however, is not enough to adequately characterize such ecosystems for forest management planning.

Most of the research concerning the application of UAS for tree management has come out of precision agriculture applications. Orchard trees are typically planted in rows with significant separation, leading to easily identifiable crown pattens. In contrast, naturally established trees do not follow such regularized patterns, making identification complicated. UAVs offer ecologists new opportunities for scale-appropriate measurements of ecological phenomena (Anderson and Gaston).

\section{METHODS}

We used LandTrendr in Google Earth Engine to extract landscape change areas with year of disturbance information. We used NAIP imagery to verify the ground pattern for the year of fire and the year of analysis. We then more closely examined the spatial distribution of saplings in 2017. Michigan Department of Natural Resource forest management shapefiles were used to verify treatments between the year of the fire and the year of observation.

\subsection{Study Site}

Unlike more economically important tree species in Michigan, jack pine forests have sustained their extent since the 1800s. The Eastern Upper Peninsula contains over 105,000 acres (or $28.6 \%$ of the type) of jack pine forest. During May and June of 2012, the Duck Lake Fire consumed nearly 22,000 acres of jack pine forest along the southern shore of Lake Superior, shown in Figure 1. The area is a mix of Michigan Department of Natural Resources Managed Units (15710 acres) and private landowners (3395 acres). The site is located meters away from Lake Superior and reaches south nearly $28 \mathrm{~km}$ across a glacial outwash moraine.

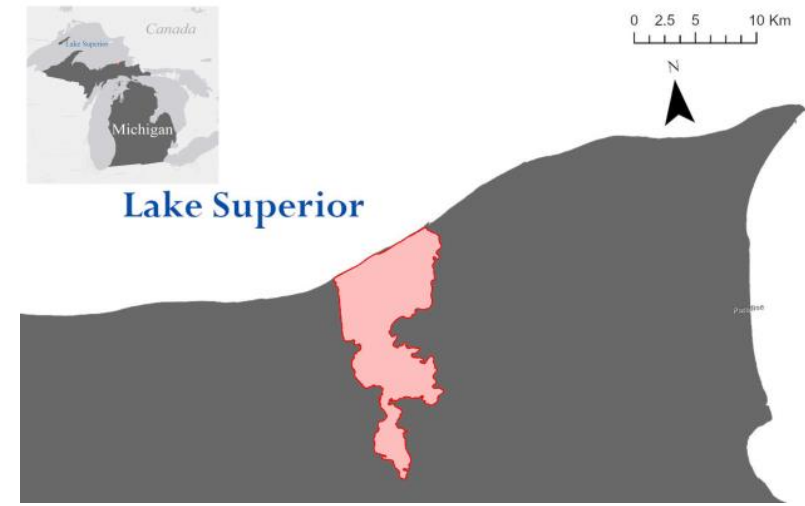

Figure 1. Study Area Overview

According to the Monitoring Trends in Burn Severity (MTBS) data (MTBS Project), the site is mostly low severity fire to moderate severity burn.

\subsection{NAIP Data}

Imagery was obtained for the burned area from the USDA Data Gateway (https://datagateway.nrcs.usda.gov/) for the years 2012 and 2016. Imagery for these years had $1 \mathrm{~m}$ spatial resolution, and four-band spectral coverage Red (R), Blue (B), Green (G), and Near Infrared (NIR). An overview of the entire classification process is provided in Figure 2.

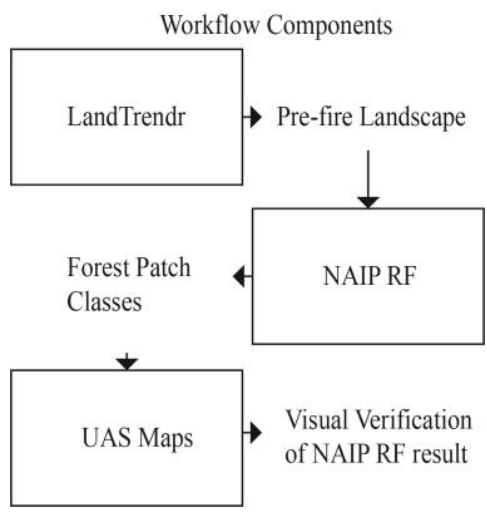

Figure 2. Overview of the three main components of analysis. (1) LandTrendr change mapping to characterize landscape history, (2) NAIP random forest classification, and (3) UAS maps for verifying a subset of the NAIP results.

\subsection{UAS Data}

UAS data was originally acquired three times throughout the 2017 summer (early, mid, and late season). Ten $100 \mathrm{~m}^{2}$ plots were established across the northern part of the burn scar. A MicaSense Red Edge camera capturing images in the Red (R), Green (G), Blue (B), Red Edge (RE) and Near Infrared (NIR) was flown on DJI Phantom 4 platform. Flights were conducted at a height of $80 \mathrm{~m}$ at a velocity of $6 \mathrm{~m} / \mathrm{s}$. The imagery was photogrammetrically processed using the Pix4Dmapper Pro version 4.4.12 resulting in RGB+NIR orthophoto mosaics. The images were georeferenced to the NAIP imagery using ArcGIS Pro 2.5.

Previous analysis of the plots had indicated that a combination of RGB with a difference between the NIR and R bands were most useful for detection of jack pine saplings (White et al.). 
The analysis was carried out on two sites of similar latitude, one with a disturbance event occurring in 1986 and the other absent of prior disturbances. The details for the LandTrendr analysis follow.

\subsubsection{UAS-OBIA}

The object-based image analysis for this project was carried out using Trimble eCognition Developer version 9. Individual analyses of the UAS data and NAIP data were conducted for the two sites. Prior to OBIA, an NDVI layer was created using each of the NAIP and UAS images.

Multiresolution segmentation using the RE band was combined with a Random Forest (RF) classifier based on texture and spectral reflectance and mean brightness. Parameters for this segmentation are available in Table 1 below.

\begin{tabular}{|l|l|}
\hline Parameter & Value \\
\hline Scale & 100 \\
\hline Shape & 0.9 \\
\hline Compactness & 0.8 \\
\hline
\end{tabular}

Table 1. Multi-resolution Image Segmentation Parameters used to segment UAS images.

A random forest classification was carried out to determine the location of jack pine saplings. The RF was trained using the mean and max brightness, mean spectral reflectance for each band, mean texture (Gray-Level Co-Occurrence Matrix (GLCM) mean) in all directions, and textural dissimilarity (GLCM dissimilarity) in all directions in order to train the classifier. Visual evaluation of the classification output was performed to clean the tree boundaries.

Accuracy assessment of the results of the classification based on presence and absence of saplings. Equations 1-3 for User Accuracy (UA), Producer Accuracy (PA), and the F-score (F).

$\mathrm{UA}=\mathrm{TP} /(\mathrm{FP}+\mathrm{TP})$

$\mathrm{PA}=\mathrm{TP} /(\mathrm{FN}+\mathrm{TP})$

$\mathrm{F}=2(\mathrm{UA} * \mathrm{PA}) / \mathrm{UA}+\mathrm{PA}$

Where $\quad \mathrm{TP}=$ True Positives

$\mathrm{FN}=$ False Negatives

\subsection{LandTrendr}

LandTrendr for Google Earth Engine (GEE) is a dense timeseries method that uses temporal segmentation of spectral trajectories to extract change information (Kennedy et al.). The availability of this algorithm via GEE has made it possible for easy implementation. The LT Change Mapper tool available from GEE produces a map containing information about the year of change, magnitude of change, duration of change event, and pre-change spectral value. Here the NBR ratio was used for the segmentation process and default values for the rest for the years 1984 to 2011, the year prior to the Duck Lake fire. This choice was made since the 2012 change wipes out spectral evidence of the past heterogeneity. The results of this analysis were then exported as a thematic array.

The most useful array data from the LandTrendr output for this study were Layer 1 (Year of Change), Layer 2 (Magnitude of
Change), and Layer 3 (Duration of Change). The results of this preliminary analysis were then compared visually to the spatial patterns apparent in pre-fire Google Earth imagery for a quick verification of the patch boundaries.

\subsection{NAIP Classification}

Object-Based Image Analysis was carried out on the NAIP data and MTBS burn severity class. Target classes were 'Disturbed Prior to 2012 Fire' and 'Not Disturbed Prior to 2012 Fire). These classes were created based on the LandTrendr findings. A total of 626 training sites were used for the classification. Of these, 103 training sites were considered disturbed prior to the 2012 event based on the LandTrendr results. These sites were visually surveyed using 2010 NAIP data to determine whether there was any indication that they had been disturbed. While visual interpretation was able to verify many of the sites, some sites with disturbances dating back to the 1980s and early 1990s were less easily verified. After this visual inspection it was determined that all sites were acceptable and all LandTrendr classification results were kept for the NAIP analysis.

Segmentation of the data was carried out using the eCognition. Multi-resolution segmentation (MRS) was used for the analysis with the settings in Table 2. The scale parameter controls spectral variation, while the shape and compactness settings are weights between the shape and color, and the compactness of the shapes generated during segmentation. Optimal segmentation parameters were determined using trial and error.

\begin{tabular}{|l|l|}
\hline Parameter & Value \\
\hline Scale & 100 \\
\hline Shape & 0.1 \\
\hline Compactness & 0.5 \\
\hline
\end{tabular}

Table 2. Segmentation parameters for the MRS process in eCognition.

Random forest (RF) classification is a machine learning algorithm has been used extensively for classification of remote sensing data. RF is ensemble learning method for classification and regression that produces multiple decision trees using bagging to select subsets of training samples and random feature selection to split them (Breiman).

\section{RESULTS}

We performed a series of analyses to evaluate whether it was possible to identify previously disturbed and/or post-fire treated sites using fine resolution imagery (NAIP $1 \mathrm{~m}$ ) with UAS imagery serving as secondary information to verify the sites had shown visual differences in the abundance of jack pine sapling survival.

\subsection{LandTrendr Change Map}

To determine the pre-fire state of disturbance, the LandTrendr algorithm was applied to the site imagery for dates between 1984 and 2011, one-year pre-fire. The map of disturbance patches identified by LandTrendr are seen in Figure 3 below. The algorithm identified change occurring in 19 of the years, but they were reclassified as 'Disturbed prior to 2012' as an aggregate. 


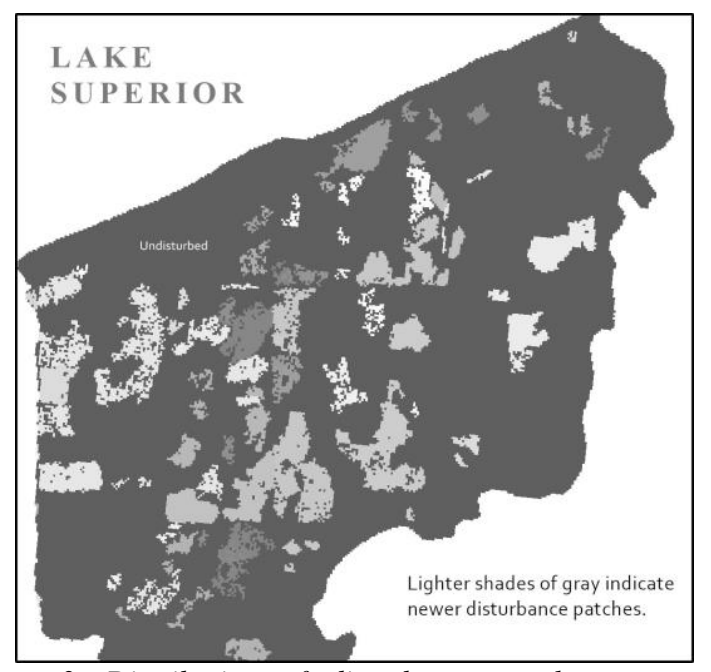

Figure 3. Distribution of disturbance patches across the northern half of the burn scar. Dark gray background indicates the boundary of the fire. Light color patches indicate pre-2012 disturbance identified by LandTrendr.

\subsection{NAIP Classification}

Using the LandTrendr results as the binary classes, a random forest classification was carried out on the NAIP data and ancillary products. The overall accuracy of this classification process considered two classes, those disturbed prior to the 2012 fire and those that were not. The classification achieved 0.53 accuracy.

The least useful of the variables was the soils data. The landscape is between $85-95 \%$ rubicon sand across the samples and the slope range for the soil units only ranged between 0 and $15 \%$. More detailed soil information should be helpful.

\section{DISCUSSION}

Understanding how past forest changes affect the revegetation of fire-disturbed landscapes is critical for developing forest management plans, understanding carbon dynamics, and predicting future forest structure. Prior research concerning the disturbance history of northern forests is commonly carried out using multispectral moderate resolution imagery, such as Landsat. The use of vegetation indices such as NBR and NDVI have proven to be useful in these cases (Kansas et al.) and our own research here suggests that the use of NDVI at finer spatial resolutions can be used to link the past disturbance patterns with future landscape patterns.

Random forest classification has been successfully applied to scenarios where historical disturbance patterns have been related to heterogenous fire-effected forest landscapes. As (Kane et al.) indicated in their research, the severity of fire and the landscapes patterns that emerge as a result of fire severity are impacted by the landscape history. In our own study, the site has been actively managed by the Michigan DNR for decades, and selective logging and replanting is common. The impact of these types of practices is clear visually from high-resolution remote sensing data, especially the UAS data we collected. Training a computer to differentiate between such states, however, is a larger challenge when working with limited multispectral data.
The use of hyperspectral and LiDAR or SfM data provide additional details about forest that would improve the classification accuracies obtained with the NAIP data. As this work is a preliminary site analysis for a continuing effort, the use of Worldview-3 data will be considered for the next iteration. Having launched in 2014 the sensor provides coverage for most of the same post-fire years as NAIP at a $0.31 \mathrm{~m}$ spatial resolution and 8 multispectral and 8 short-wave infrared bands, the spectral resolution it may outperform the NAIP data. In addition to more robust spectral coverage, the use of highresolution forest structure data would also improve the classification results. Previous research has shown that SfM cannot match the results provided by land-based LiDAR techniques (Wallace et al.). However, the cost of LiDAR scanning from ground or air is still expensive and improvements to photogrammetric techniques such as SfM would be beneficial for management use.

This research led to the development of a workflow that can be used to integrate high-resolution remote sensing data, in the form of NAIP and UAS, for mapping landscape history impacts on fire-impacts. The relationship between disturbance history and fire severity is one that is well-suited to high-resolution data and benefits not only the scientific understanding of forest cycles such as carbon cycling, but also informs management decision-making.

\section{CONCLUSIONS}

Improvements in technological abilities and access to higherresolution data are improving our ability to understand ecological processes and spatial patterns of forests. This research demonstrated the integration of high-resolution aerial photography with UAV -based sensors to identify and spatially characterize the distribution of jack pine saplings in an early successional environment.

Prior to this study, limited research has been conducted to examine the potential for UAS to map individual seedling and sapling characteristics in natural environments due to the diminutive size of these plants. Such small plants are also often overlooked by scientists. This analysis found that the using submeter multispectral imagery including the Near-Infrared channel can characterize the density of their distribution based on a relationship between NAIP spectral data and pre-fire disturbance. Future work should consider a larger area of UAS imagery, preferably captured at the same time as NAIP data or a multispectral satellite product, such as Worldview-3. Also, further work should be conducted concerning the potential relationship between pre-fire condition and post-fire sapling survivability.

\section{REFERENCES}

Anderson, Karen, and Kevin J. Gaston. "Lightweight Unmanned Aerial Vehicles Will Revolutionize Spatial Ecology." Frontiers in Ecology and the Environment, vol. 11, no. 3, Wiley Online Library, 2013, pp. 13846.

Breiman, Leo. "Random Forests." Machine Learning, vol. 45, no. 1, Oct. 2001, pp. 5-32, doi:10.1023/A:1010933404324.

Buters, Todd, et al. "Seed and Seedling Detection Using Unmanned Aerial Vehicles and Automated Image Classification in the Monitoring of Ecological 
Recovery." Drones, vol. 3, no. 3, Multidisciplinary Digital Publishing Institute, 2019, p. 53.

Corace, R. Gregory, et al. "Upland Sandpiper: A Flagship for Jack Pine Barrens Restoration in the Upper Midwest?" Ecological Restoration, vol. 34, no. 1, 2016, pp. 49-60.

Fernandez-Manso, Alfonso, et al. "Burn Severity Influence on Post-Fire Vegetation Cover Resilience from Landsat MESMA Fraction Images Time Series in Mediterranean Forest Ecosystems." Remote Sensing of Environment, vol. 184, 2016, pp. 112-23, doi:https://doi.org/10.1016/j.rse.2016.06.015.

Frazier, Ryan J., et al. "Analyzing Spatial and Temporal Variability in Short-Term Rates of Post-Fire Vegetation Return from Landsat Time Series." Remote Sensing of Environment, vol. 205, Elsevier, 2018, pp. 32-45.

Hicke, Jeffrey A., et al. "Postfire Response of North American Boreal Forest Net Primary Productivity Analyzed with Satellite Observations." Global Change Biology, vol. 9, no. 8, Wiley Online Library, 2003, pp. 114557.

Kane, Van R., et al. "Mixed Severity Fire Effects within the Rim Fire: Relative Importance of Local Climate, Fire Weather, Topography, and Forest Structure." Forest Ecology and Management, vol. 358, Dec. 2015, pp. 62-79, doi:10.1016/j.foreco.2015.09.001.

Kansas, John, et al. "Using Landsat Imagery to Backcast Fire and Post-Fire Residuals in the Boreal Shield of Saskatchewan: Implications for Woodland Caribou Management." International Journal of Wildland Fire, vol. 25, no. 5, CSIRO, 2016, pp. 597-607.

Kennedy, Robert E., et al. "Implementation of the LandTrendr Algorithm on Google Earth Engine." Remote Sensing, vol. 10, no. 5, Multidisciplinary Digital Publishing Institute, 2018, p. 691.

Lydersen, Jamie M., and Brandon M. Collins. "Change in Vegetation Patterns over a Large Forested Landscape Based on Historical and Contemporary Aerial Photography." Ecosystems, vol. 21, no. 7, Springer, 2018, pp. 1348-63.

Matese, Alessandro. Editorial for the Special Issue "Forestry Applications of Unmanned Aerial Vehicles (UAVs)., Multidisciplinary Digital Publishing Institute, 2020.

Meng, Ran, et al. "Measuring Short-Term Post-Fire Forest Recovery across a Burn Severity Gradient in a Mixed Pine-Oak Forest Using Multi-Sensor Remote Sensing Techniques." Remote Sensing of Environment, vol. 210, Elsevier, 2018, pp. 282-96.

MTBS Project. MTBS Data Access: Fire Level Geospatial Data. USDA Forest Service/U.S. Geological Survey, July 17AD, http://mtbs.gov/direct-download.

Pádua, L., et al. "POST-FIRE FORESTRY RECOVERY MONITORING USING HIGH-RESOLUTION MULTISPECTRAL IMAGERY FROM UNMANNED AERIAL VEHICLES." International Archives of the Photogrammetry, Remote Sensing \& Spatial Information Sciences, 2019.

Potter, Christopher, et al. "Analysis of Sapling Density Regeneration in Yellowstone National Park with Hyperspectral Remote Sensing Data." Remote Sensing of Environment, vol. 121, Elsevier, 2012, pp. 61-68.

Spaulding, Susan E., and David E. Rothstein. "How Well Does Kirtland's Warbler Management Emulate the Effects of Natural Disturbance on Stand Structure in Michigan Jack Pine Forests?" Forest Ecology and Management, vol. 258, no. 11, 2009, pp. 2609-18.
Tucker, Madelyn M., and Daniel M. Kashian. "Pre-Fire Forest Remnants Affect Post-Fire Plant Community Structure and Composition." Forest Ecology and Management, vol. 408, Elsevier, 2018, pp. 103-11.

Wallace, Luke, et al. "Assessment of Forest Structure Using Two UAV Techniques: A Comparison of Airborne Laser Scanning and Structure from Motion (SfM) Point Clouds." Forests, vol. 7, no. 3, Multidisciplinary Digital Publishing Institute, 2016, p. 62.

White, Raechel A., et al. "UAS-GEOBIA Approach to Sapling Identification in Jack Pine Barrens after Fire." Drones, vol. 2, no. 4, 2018, p. 40. 